\title{
Mirvetuximab Soravtansine
}

National Cancer Institute

\section{Source}

National Cancer Institute. Mirvetuximab Soravtansine. NCI Thesaurus. Code C102566.

An immunoconjug ate consisting of the humanized monoclonal antibody M9346A against folate receptor 1 (FOLR1) conjug ated, via the disulfide-containing cleavable linker sulfoSPDB, to the cytotoxic maytansinoid DM4, with potential antineoplastic activity. The antiFOLR1 monoclonal antibody moiety of mirvetuximab soravtansine targets and binds to the cell surface antigen FOLR1. After antibody-antigen interaction and internalization, the immunoconjug ate releases DM4, which binds to tubulin and disrupts microtubule assembly/disassembly dynamics, thereby inhibiting cell division and cell growth of FOLR1expressing tumor cells. FOLR1, a member of the folate receptor family is overexpressed on a variety of epithelial-derived cancer cells. The sulfo-SPDB linker prevents cleavage in the bloodstream and may improve this agent's efficacy in multidrug resistant tumor cells. 